\title{
Estudio de la anatomía del xilema secundario de seis especies del género Citrus
}

cultivadas en Costa Rica

\section{Resumen}

En Costa Rica, algunas especies del género Citrus son plantadas para la producción del fruto o como árboles de servicio en cultivos de café (Coffea spp). Sin embargo, el aprovechamiento de estas plantaciones se limita solamente al fruto, por lo que al llegar al final de su vida útil, las partes leñosas del árbol son despreciadas, a pesar de ser una fuente importante de biomasa para la producción de energía. En el presente trabajo se estudió la anatomía del xilema secundario de seis especies del género Citrus: C. aurantifolia (limero), C. limettioides (limón dulce), C. reticula (mandarina), C. paradisi (toronja), C. sinensis (naranja) y una variedad, C. sinensis var. Washington (naranja injertada), mediante la descripción anatómica (macroscópica y microscópica) de la madera de estas especies, con el objetivo de aportar información para la utilización del fuste. Para ello, muestras del fuste fueron descritas utilizando las normas internacionales de descripción de madera. Los resultados mostraron que la madera de las especies de Citrus evaluadas presentan una densidad seca menor de $500 \mathrm{~kg} / \mathrm{m}^{3}$,

\author{
Oxana Brenes-Angulo ${ }^{1}$ \\ David Reyes-Cordero ${ }^{1}$ \\ Roger Moya-Roque ${ }^{1}$
}

\begin{abstract}
Study of secondary xylem of six species of the genus Citrus used in Costa Rica. Some of the species of the genus Citrus are planted in Costa Rica for fruit production or as shadow trees in coffee (Coffea sp) plantations. However, the exploitation of these plantations is limited only to the fruit, leaving many parts of the tree without a use, which they can be an important biomass source for energy production. In the present work, it was studied the anatomy of the secondary xylem of six species of the genus Citrus: C. aurantifolia (limero), C. limettioides (sweet lemon), C. reticula (tangerine), C. paradisi (grapefruit), C. sinensis (orange) and a variety, C. sinensis var. Washington Navel (hybrid orange) by the anatomical description (macroscopic and microscopic) of their wood, with the aim of providing information for a future process of their trunk's use. For this, there were taken samples of the trees' trunks, which were described using international standards of wood description. The results showed that different Citrus species had a dry density wood less than $500 \mathrm{~kg} / \mathrm{m} 3$, a fine to medium-
\end{abstract}


textura fina a mediana, color amarrillo a amarrillo pálido, sin veteado, sabor u olor característico. Así mismo, mostraron porosidad difusa y poros solitarios, pequeños y de alta abundancia. Se observaron radios de tipo homocelulares, de 1 a 3 células de ancho y de 10 a 25 células en altura. Para el parénquima axial, se presentó una gran variedad de tipos, que van desde paratraqueal hasta el apotraqueal, y bandas de parénquima formando anillos falsos o bien anillos anuales. La presencia de canales traumáticos y cristales en madera también estuvieron presentes en todas las especies. En general, se evidencia que la anatomía de la madera de las seis especies estudiadas presenta poca diferencia entre ellas, por lo que su posible identificación a este nivel se dificulta.

Palabras claves: Citrus, utilización de la madera, propiedades de madera, frutales, sombra de café.

\section{Introducción}

En general, el género Citrus presenta árboles de tipo perenne, de mediana altura y con diámetro de fuste muchas veces mayor a $10 \mathrm{~cm}$ (Sánchez 2005). Las numerosas especies de este género provienen de las zonas tropicales y subtropicales de Asia y del archipiélago Malayo. En Costa Rica, la producción de cítricos, principalmente naranja, ha aumentado significativamente en las regiones de Sarapiquí, Grecia, Los Chiles, San Carlos y Upala, entre otros, además de las áreas tradicionales de explotación ubicadas en los cantones de Acosta, Mora, Puriscal, Aserrí, Atenas, Pérez Zeledón y Alajuela (Molina 1999).

En el país, este tipo de árboles eran plantados en asocio con el cultivo del café (Coffea sp), formando parte de un sistema agroforestal con especies maderables y especies de crecimiento rápido para sombra (Corrales 1997). En los últimos años, se han establecido plantaciones puras, principalmente de naranja, en grandes extensiones para su uso como fruta para consumo fresco, procesamiento como jugos y exportación (Corrales 1997). El área citrícola pasó de aproximadamente 5000 ha en 1989 a más de 18000 ha en 1993 (Brenes y Arauz 1994).

Se estima que los Citrus presentan un periodo de producción de 20 años, posteriormente los árboles son cortados. Durante esa eliminación, ya sea en plantaciones comerciales o en los mismos cafetales, los árboles son seccionados en pequeñas longitudes y se asigna un espacio para depositar toda la biomasa (hojas, fustes y ramas), para que ocurra su degradación natural. Este material, en especial el fuste, representa una excelente opción para su aprovechamiento, por la gran cantidad de material disponible. Por ejemplo, se ha estimado que en promedio, un árbol de Citrus presenta sized texture, a pale yellow to yellow color, and no veined, flavor or scent. Also, the wood presented diffuse - porous and high abundance of solitary and small pores. There were observed homocellular rays, with a 1-3 cell width and 10-25 cell height. For axial parenchyma, it presented a wide variety of types, ranging from paratracheal to apotracheal, and some parenchyma bands forming false or either annual rings. The presence of traumatic canals and crystals in the species' wood were also determined. Generally it was evident that the anatomy of the six studied species presented little difference between them, which it is why the difficultness of their possible identification.

Key words: Citrus, wood use, wood properties, fruit trees, coffea shadow.

una materia seca de aproximadamente $25 \mathrm{~kg}$ (Mattos et al. 2003).

Sin embargo, para dar un aprovechamiento industrial a los Citrus es necesario conocer sobre la anatomía de su xilema, puesto que su estudio permite establecer los posibles usos y futuros comportamientos en los procesos de manufactura. En el presente trabajo se describe la anatomía del xilema secundario y características generales de seis especies del género Citrus (C. aurantifolia, C. limettioides, C. reticula, C. paradisi, C. sinensis y C. sinensis var. Washington) de mayor uso en Costa Rica. Esto permitirá aportar información en caso de iniciar un proceso de utilización del fuste en usos altamente necesitados en países en desarrollo, sea como la utilización de su madera o bien como fuente de biomasa para la producción de energía. Así mismo esta información permitirá establecer las diferencias anatómicas entre especies y así lograr en su futuro una posible identificación.

\section{Materiales y Métodos}

Las muestras se tomaron de árboles ubicados en plantaciones de café (Coffea spp.), en el valle de Orosí, Cartago, Costa Rica (09 43' 51' Latitud Norte y $83^{\circ} 45^{\prime}$ 46" Longitud Oeste), a excepción de C. sinensis, que estaba creciendo en una plantación pura en la zona de Naranjo, Alajuela, Costa Rica (1006'23" Latitud Norte y 84²3'23" Longitud Oeste). Los árboles seleccionados eran individuos maduros y sin presencia de daños por insectos u hongos.

Se utilizó un árbol por cada especie, debido a que en ningún caso fue posible muestrear más individuos, por tratarse de frutales en plena producción. De cada árbol, 
se extrajo una muestra representativa de una pequeña sección del fuste de aproximadamente $30 \mathrm{~cm}$ de longitud y a una altura de aproximadamente $15 \mathrm{~cm}$ sobre el nivel del suelo. El material obtenido por cada especie fue llevado al Laboratorio de Anatomía de la Madera del Instituto Tecnológico de Costa Rica, donde se preparó una muestra de $15 \mathrm{~cm}$ de largo, $12 \mathrm{~mm}$ de espesor y $7,5 \mathrm{~cm}$ en ancho, por cada individuo, para la descripción de las características generales de la madera, y una muestra más pequeña $(1 \mathrm{~cm} \times 1 \mathrm{~cm} \times 1 \mathrm{~cm})$, para el estudio anatómico.

Determinación de las propiedades generales de la madera: Las propiedades generales determinadas fueron: color, brillo, olor, sabor, textura, dirección del grano y densidad de la madera. El color fue determinado por medio de las tablas de Munsell para suelos (Mac Corporation 1994). En el resto de las características, a excepción de la densidad, fueron utilizados los criterios de la Comisión Pan-Americana de Normas Técnicas (COPANT 1974) y las "Normas e Procedimentos em Estudos da Anatomía da Madeira" (IBAMA 1992). La densidad fue determinada por el peso de la muestra y volumen con las dimensiones de ancho, largo y espesor de la pieza.

Descripción anatómica de la madera: En la descripción de la estructura macroscópica fueron considerados como referencia los términos establecidos por Espinoza y León (2001). Además, fueron consultados los términos usados por la Asociación Internacional de Anatomista de la Madera (IAWA 1989), los criterios de la Comisión Pan-Americana de Normas Técnicas (COPANT 1974) y las Normas y Procedimientos en Estudios de Anatomía de Madera (IBAMA 1992). La observación de la madera se realizó con la ayuda de una lupa de 10X, y se estudiaron los tres elementos fundamentales de ella (vasos, parénquima y radios) y la formación de anillos de crecimiento. Los diferentes elementos anatómicos observados, tomando en consideración las normas anteriores, fueron catalogados de la siguiente manera:

Poros: se observó el tamaño de ellos de acuerdo a una visibilidad con aumento de 10X (visible, ligeramente visible y no visible); el tipo de porosidad (circular, semicircular y difuso); el agrupamiento (poros solitarios o múltiples); la disposición (cadenas radiales, tangenciales o déndrica) y la presencia de gomas o tílides por los contenidos en el lumen de los poros.

Parénquima axial: se observó si éste era distinguible, mediante la diferenciación en los tonos del color alrededor de los vasos o en general, de la sección transversal de la muestra en estudio. Esto permitió definir los tipos de parénquima: paratraqueal, apotraqueal y en bandas. En el parénquima paratraqueal se establecieron las siguientes categorías: escaso, vasicéntrico, unilateral, aliforme (fino o grueso) y aliforme confluente. El parénquima apotraqueal fue categorizado como apotraqueal difuso y en agregados. El parénquima en bandas se clasificó como bandas abundantes o escasas, finas o gruesas, marginales, escalariforme o reticulada.

Parénquima radial: se determinó si eran visibles o no en la sección transversal, el tamaño (grueso o finos) y la frecuencia (abundante, mediana abundancia y poco abundante). Posteriormente, en el plano tangencial, se observó si se presentaban estratificados o no estratificados.

Otros elementos anatómicos: en la observación de la madera también se estableció la existencia de otros elementos anatómicos que pudieran ser observados a nivel macroscópico, tales como la presencia de floema incluso, conductos gomíferos o canales traumáticos. También se evaluó la marcación de los anillos en la sección transversal (presencia - ausencia), y en caso de estar presentes se determinó la característica que los define (presencia de una banda marginal, concentración de fibras o presencia de porosidad circular o semicircular); además fueron preparados cortes histológicos para observar microscópicamente la marcación del anillo.

La descripción microscópica fue realizada por medio de cortes histológicos de madera sobre láminas permanentes y maceración del xilema. La maceración y la preparación de dichos cortes fueron hechas siguiendo la metodología propuesta por Johansen (1940) y Sass (1951). Para la descripción de la anatomía del xilema se utilizaron criterios de IAWA(1989). En aquellos casos donde fue necesario medir una variable específica, se utilizó un microscopio con una cámara digital y las imágenes fueron analizadas con el software IMAGE TOOL ${ }^{\circledR}$.

\section{Resultados y Discusión}

\section{Características generales}

Las especies de Citrus evaluadas no presentaron una marcación entre la albura y el duramen, excepto en $C$. paradisi, en la cual se observó una ligera marcación entre ambos tipos de madera. En todas las especies, la madera se catalogó de color amarrillo a amarrillo pálido, excepto en C. paradisi en condición seca, que presentó una albura de color blanco con tendencia a amarillo pálido y un duramen de color marrón claro (Figura 1).

En lo referente a las características organolépticas, la textura de la madera de las especies evaluadas se clasificó de media a fina, no se presentó sabor ni olor característicos, el brillo se catalogó de moderado a brillante y no se apreció veteado. En general, se observó madera con grano recto, solamente interrumpido por la presencia de nudos. 


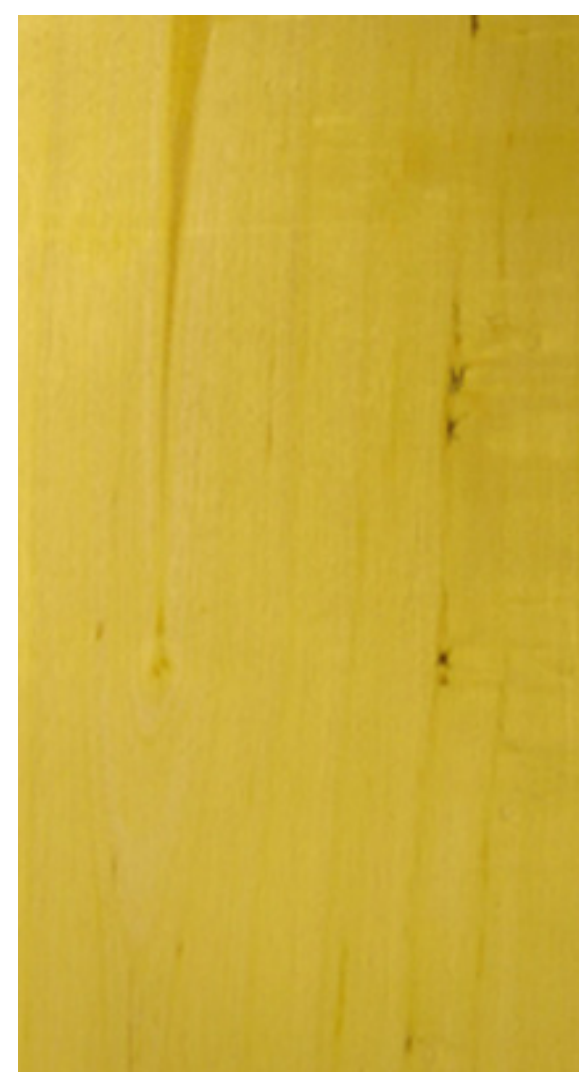

Citrus aurantifolia

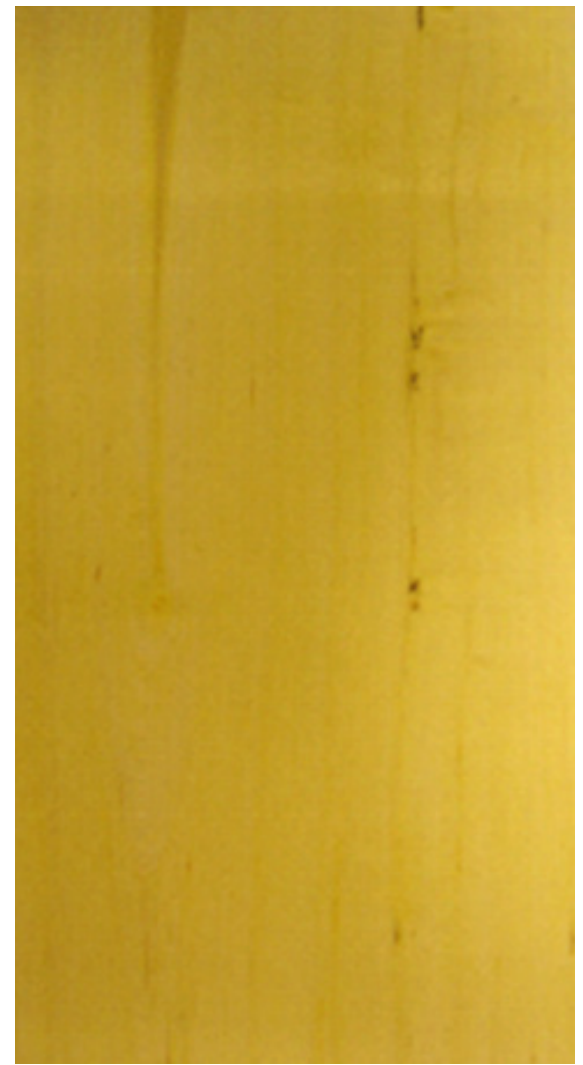

Citrus sinensis

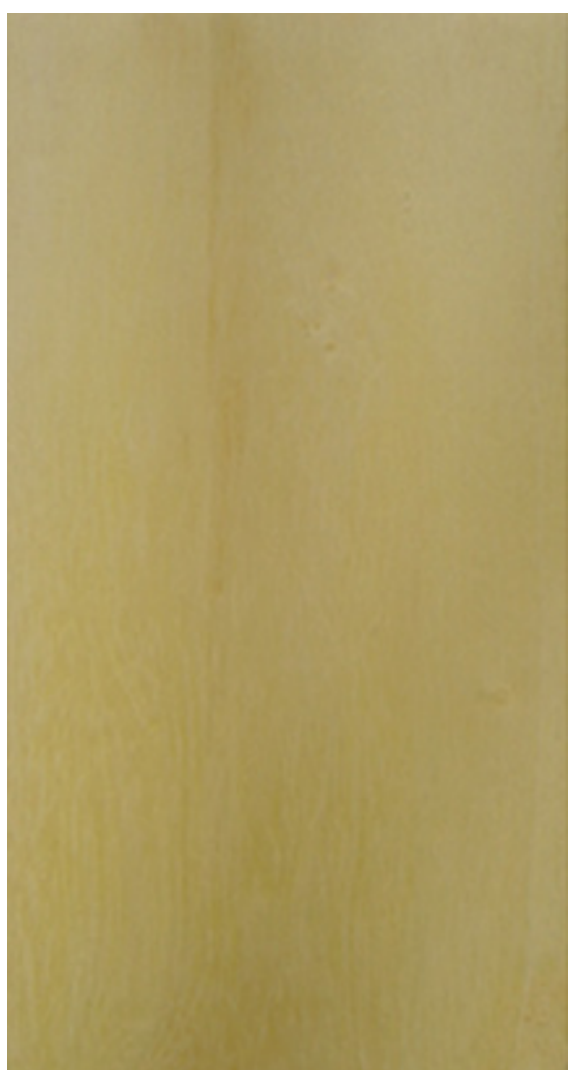

Citrus limettioides

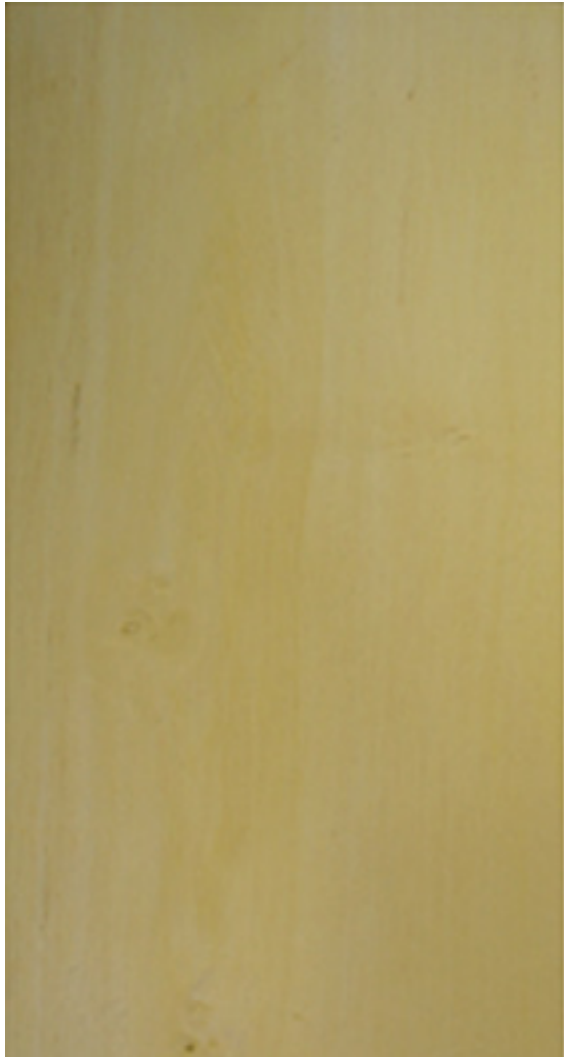

Citrus sinensisvar washington
Citrus reticula

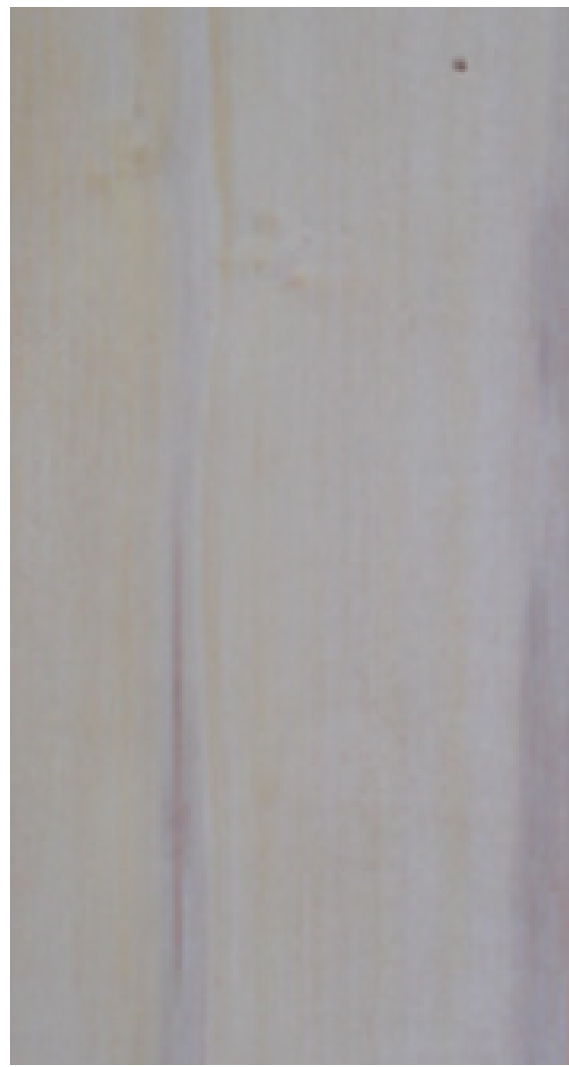

Citrus paradidi macfad

Figura 1. Vista tangencial de la madera de seis especies del género Citrus cultivadas en Costa Rica. 
Cuadro 1. Propiedades generales de la madera de seis especies del género Citrus cultivadas en Costa Rica.

\begin{tabular}{|c|c|c|c|c|c|c|}
\hline Característica & C. aurantifolia & C. limettioides & C. reticula & C. sinensis & $\begin{array}{l}\text { C. sinensis var. } \\
\text { Washington }\end{array}$ & C. paradisi \\
\hline Duramen/albura & No hay marcación & No hay marcación & No hay marcación & $\begin{array}{l}\text { No hay mar- } \\
\text { cación }\end{array}$ & No hay marcación & Hay ligera marcación \\
\hline Color de duramen & Amarillo 5Y 8/6 & $\begin{array}{l}\text { Amarillo pálido } \\
5 Y 8 / 4\end{array}$ & $\begin{array}{l}\text { Amarillo pálido } \\
5 Y 8 / 4\end{array}$ & Amarillo 5Y 8/6 & $\begin{array}{l}\text { Amarillo pálido } \\
5 Y 8 / 3\end{array}$ & $\begin{array}{l}\text { Marrón claro } \\
\text { 10YR 8/2 }\end{array}$ \\
\hline Color de albura & Amarillo 5Y 8/6 & $\begin{array}{l}\text { Amarillo pálido } \\
5 Y 8 / 4\end{array}$ & $\begin{array}{l}\text { Amarillo pálido } \\
5 Y 8 / 4\end{array}$ & Amarillo 5Y 8/6 & $\begin{array}{l}\text { Amarillo pálido } \\
5 Y 8 / 3\end{array}$ & $\begin{array}{l}\text { Blanco a amarillo } \\
\text { pálido } 10 \text { YR } 8 / 1 \text { y } \\
\text { 10YR } 8 / 2\end{array}$ \\
\hline Lustre & $\begin{array}{l}\text { Bajo y ligero en la } \\
\text { cara radial }\end{array}$ & $\begin{array}{l}\text { Bajo y ligero en la } \\
\text { cara radial }\end{array}$ & $\begin{array}{l}\text { Bajo y ligero en la } \\
\text { cara radial }\end{array}$ & Ligero & $\begin{array}{l}\text { Bajo y ligero en la } \\
\text { cara radial }\end{array}$ & De regular a brillante \\
\hline Olor & Característico & Característico & Característico & Característico & Característico & Imperceptible \\
\hline Sabor & Imperceptible & Imperceptible & Imperceptible & Poco amargo & Imperceptible & Imperceptible \\
\hline Textura & Media & Media & Media & Fina a media & Media & Fina \\
\hline Densidad $\left(\mathrm{g} / \mathrm{cm}^{3}\right)$ & 0,78 & 0,44 & 0,52 & 0,49 & 0,49 & 0,75 \\
\hline Clasificación & Pesada & Moderada & Moderada & Moderada & Moderada & Pesada \\
\hline Grano & $\begin{array}{l}\text { Recto a ligeramente } \\
\text { inclinado }\end{array}$ & $\begin{array}{l}\text { Recto a ligeramente } \\
\text { inclinado }\end{array}$ & $\begin{array}{l}\text { Recto a ligera- } \\
\text { mente inclinado }\end{array}$ & $\begin{array}{l}\text { Recto a muy } \\
\text { recto }\end{array}$ & $\begin{array}{l}\text { Recto a ligeramente } \\
\text { inclinado }\end{array}$ & $\begin{array}{l}\text { Ondulado y } \\
\text { entrecruzado }\end{array}$ \\
\hline $\begin{array}{l}\text { Marcación de } \\
\text { anillos }\end{array}$ & Poco definidos & Poco definidos & Poco definidos & Indistintos & Definidos & Definidos \\
\hline
\end{tabular}

En relación a la densidad de la madera seca al aire, cuatro especies (C. limettioides, C. reticula, C. sinensis y C. sinensis var. Washington) presentaron valores similares entre ellas, con un promedio de 0,49 a $0,51 \mathrm{~kg} /$ $\mathrm{m}^{3}$; en tanto que $C$. paradisi y $C$. aurantifolia mostraron una densidad mayor, de $0,75 \mathrm{~kg} / \mathrm{m}^{3}$ y $0,78 \mathrm{~kg} / \mathrm{m}^{3}$, respectivamente (Cuadro 1).

Los resultados encontrados y detallados en el Cuadro 1, sugieren que estas propiedades organolépticas no son buenos indicadores para identificar los tipos de xilema de las seis especies de Citrus estudiadas. No obstante, la densidad de la madera podría ser un parámetro para separar dos de estas especies de las cuatro restantes, puesto que las muestras de C. aurantifolia y C. paradisi presentaron una densidad muy superior al resto de especies. En cuanto al color de la madera, C. paradisi presentó un color blancuzco, mientras que $C$. aurantifolia y $C$. sinensis presentaron un amarillo más intenso que las restantes tres especies, lo que puede ayudar a identificar estas especies, a pesar de ser una variable subjetiva.

\section{Marcación de anillos de crecimiento}

Aunque en todas las especies se observó una tendencia a formar anillos de crecimiento, fue frecuente la presencia de anillos falsos (no hay formación de un anillo en toda la circunferencia del árbol). Dicho resultado confirma lo encontrado por Abrems y Hock (2006) para C. sinensis creciendo en la parte norte de Costa Rica, quienes no observaron la presencia de anillos de crecimiento.

Los anillos de crecimiento observados a nivel macroscópico presentaron una línea de parénquima de color clara. En tanto que, microscópicamente, se observó una orientación tangencial de los poros, aunque sin porosidad de tipo anular o semianular. Así mismo, se observó que en las especies con presencia de bandas marginales de parénquimas, estas estaban compuestas por 2 a 3 células de ancho, e interrumpidas, lo que producía el anillo falso. La Figura 2 ilustra la marcación de anillos en la especie $C$. reticula, donde se puede apreciar el anillo de crecimiento (Figura 2a), los anillos falsos (Figura 2b) y la cantidad de células de parénquima en ancho que conforman la banda marginal de parénquima (Figura 2c).

Se debe recordar que los árboles o arbustos plantados en plantaciones de café (como en el caso de los Citrus utilizados en el presente estudio) son sometidos a podas o corta total de la copa y posterior manejo de rebrotes, con el fin de brindar luz al cafeto. Estas prácticas dan como resultado un crecimiento intermitente en el árbol, provocando irregularidades en los anillos de crecimiento, que inducen a la aparición de falsos anillos de crecimiento (Espinoza y León 2001).

Al igual que las variables analizadas, la presencia de anillos de crecimiento no puede utilizarse como elemento determinante para separar entre sí las especies en estudio.

\section{Descripción microscópica de la madera}

Vasos: En términos generales, los vasos no son visibles a simple vista, por lo que se tienen que observar con una lupa con un aumento mínimo de 10X (Cuadro 2). 


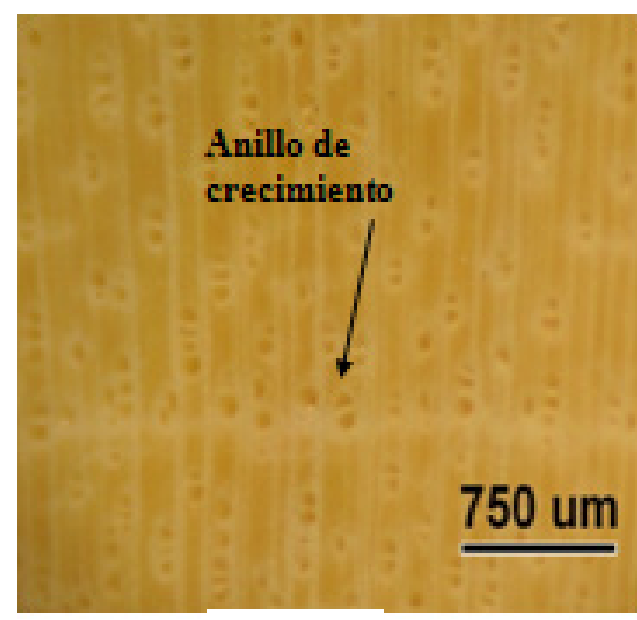

(2a)

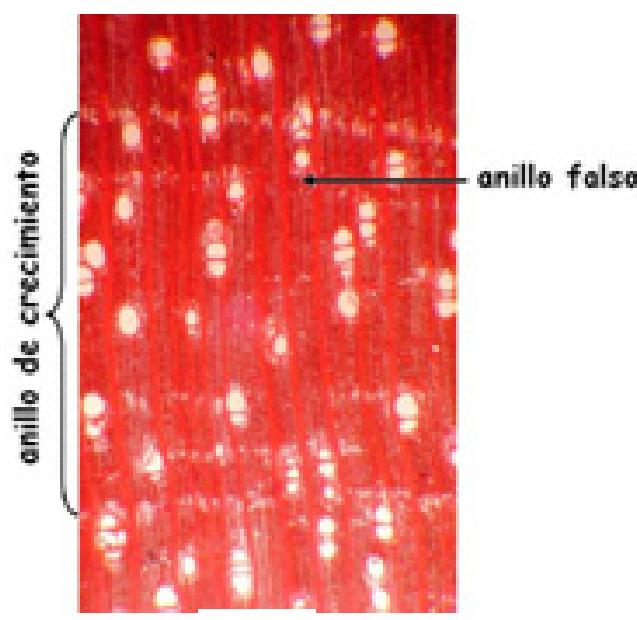

(2b)

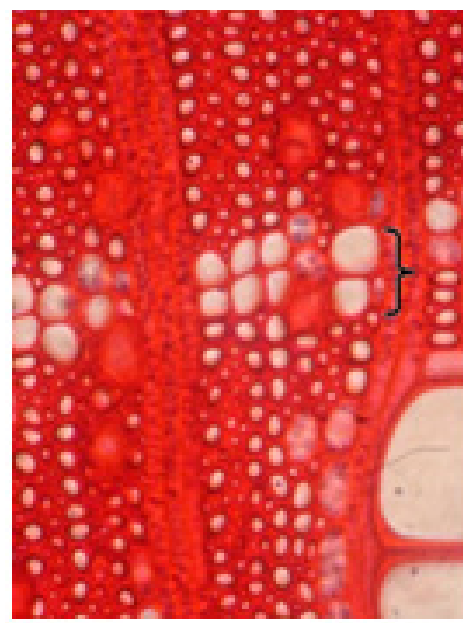

(2c)

Figura 2. Presencia y formación de los anillos de anillos de crecimiento en C. reticula: 2a) anillo de crecimiento, 2b) anillos falsos y 2c) células de parénquima que conforman la banda marginal de parénquima.

La porosidad observada en las seis especies de Citrus evaluadas fue de tipo difusa, aunque en algunos casos fue posible confundirla con porosidad semianular, debido a que los poros estaban unidos tangencialmente, en especial en $C$. sinensis var. Washington. De igual manera, los poros fueron muy similares en las seis especies, tanto en el diámetro y la longitud, como en la forma de las células (Cuadro 2 y Figura 3), aunque con diferencias en la frecuencia y porcentaje de poros solitarios y múltiples. La mayor cantidad de poros solitarios fue encontrado en C. paradisi, seguidos por C. aurantifolia y C. sinensis var. Washington.

Se encontró que las seis especies estudiadas presentaron puntuaciones de tipos hexagonales o redondeadas, dispuestas de forma alterna y de mediana abundancia. La presencia de gomas dentro de los vasos también fue común en todas las especies. Sin embargo, se observó una mayor cantidad de este tipo de sustancias en $C$. aurantifolia, C. sinensis y $C$. sinensis var. Washington. La presencia de canales traumáticos estuvo solamente presente en C. aurantifolia, permitiendo ser la características principal para su identificación (Cuadro 2).

Fibras: La pared celular de las fibras osciló entre 3,17 y $4,51 \mu \mathrm{m}$, con longitudes que no superaron $1 \mathrm{~mm}$, por lo que se consideraron como fibras cortas, siguiendo la clasificación de IAWA (1989). Las dimensiones transversales (diámetro de la fibra y diámetro del lumen) fueron muy similares en todas las especies (Cuadro 3), por lo que la utilización de esta característica es de poca utilidad para la identificación de las especies de Citrus. No obstante, en C. paradisi se observó ausencia de cristales, a diferencia del resto de las especies, en donde estos si estuvieron presentes y clasificados como romboidales escasos.
Parénquima axial: La disposición, distribución y cantidad de células fueron los elementos anatómicos de mayor variación dentro de los Citrus evaluados (Cuadro 4). Las seis especies presentaron parénquima paratraqueal y apotraqueal. Sin embargo, la cantidad de células del parénquima varió entre ellas, por ejemplo, C. aurantifolia, $C$. reticula y $C$. sinensis var. Washington mostraron mayor cantidad de parénquima alrededor de los vasos, en comparación con $C$. limettioides y $C$. sinensis (Figura 3). Por otra parte, C. sinensis var. Washington presentó la menor cantidad de parénquima apotraqueal, mientras que $C$. sinensis la mayor cantidad, con 4 a 5 células en cada cúmulo, casi similar a parénquima aprotraqueal en agregados (Figura 3).

Parénquima radial: Para las seis especies de Citrus evaluadas, el parénquima radial presentó características muy similares. Sin embargo, con $C$. sinensis var. Washington presentó los radios ligeramente más anchos y más largos; mientras que $C$. sinensis mostró los radios más pequeños (Cuadro 5 y Figura 4). Con respecto a la composición, los radios fueron homogéneos en todas las especies evaluadas (Cuadro 5).

\section{Conclusiones}

Las especies estudiadas presentan madera de densidad moderada, por lo que tienen amplias posibilidades de ser utilizadas en diferentes productos, para diámetros de fuste apropiados.

No se presenta diferenciación entre albura y duramen para ninguna de las seis especies de Citrus estudiadas; además, presentan propiedades organolépticas similares, dificultando la identificación de cada especie basado en esta característica. 


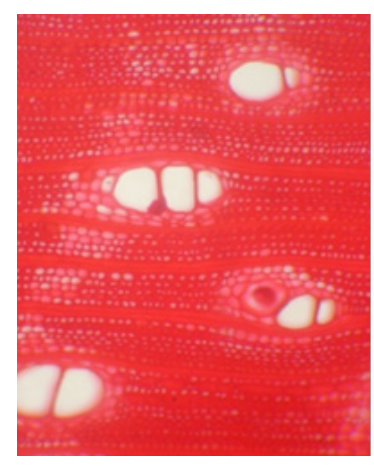

Citrus aurantifolia

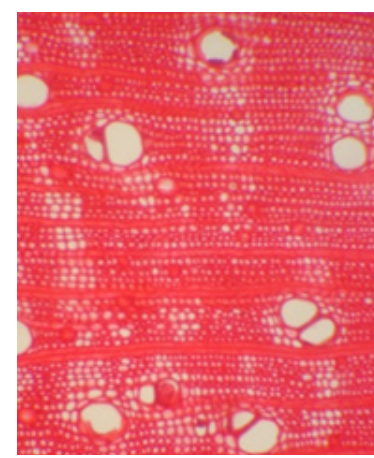

Citrus sinensis

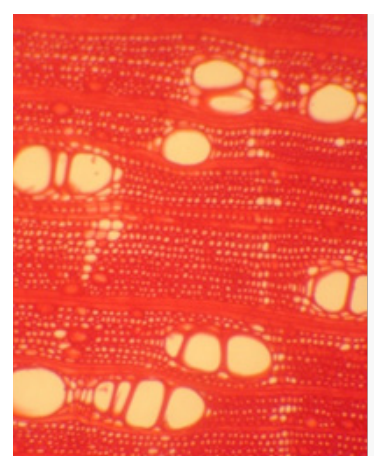

Citrus limettioides

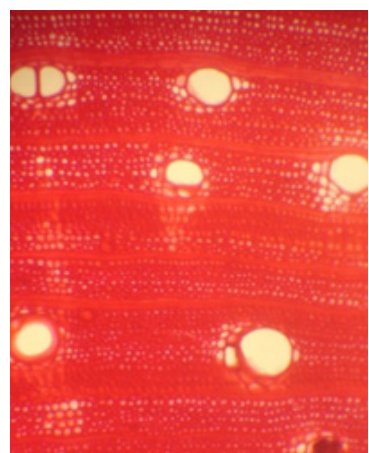

Citrus

sinensis var. Washington

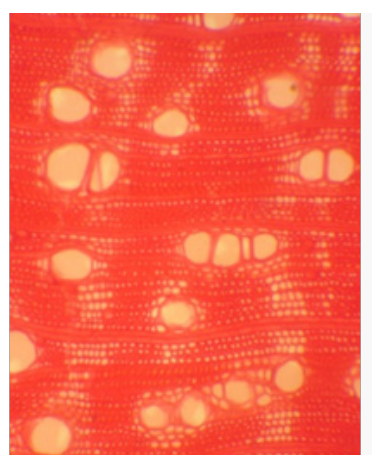

Citrus reticula

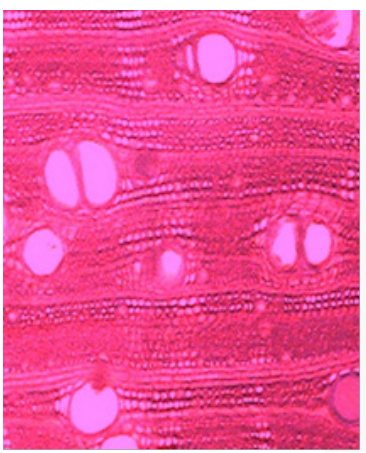

Citrus paradisi

Figura 3. Corte transversal mostrando los diferentes tipos de parénquima axial observados en seis especies del género Citrus cultivadas en Costa Rica.
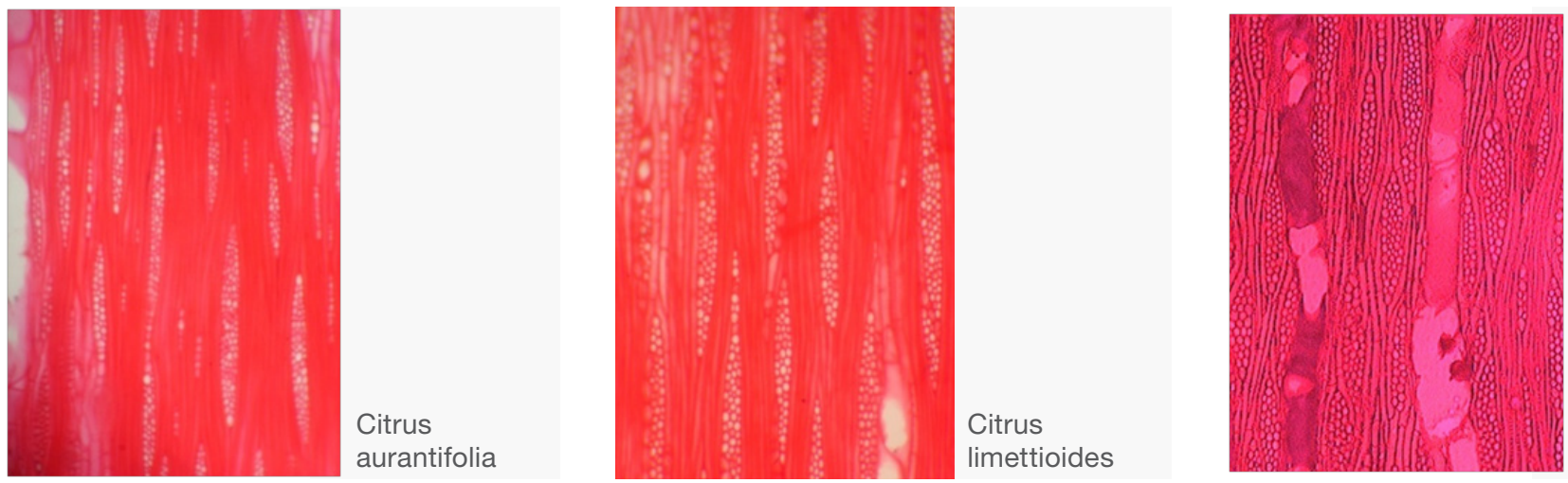

Citrus reticula
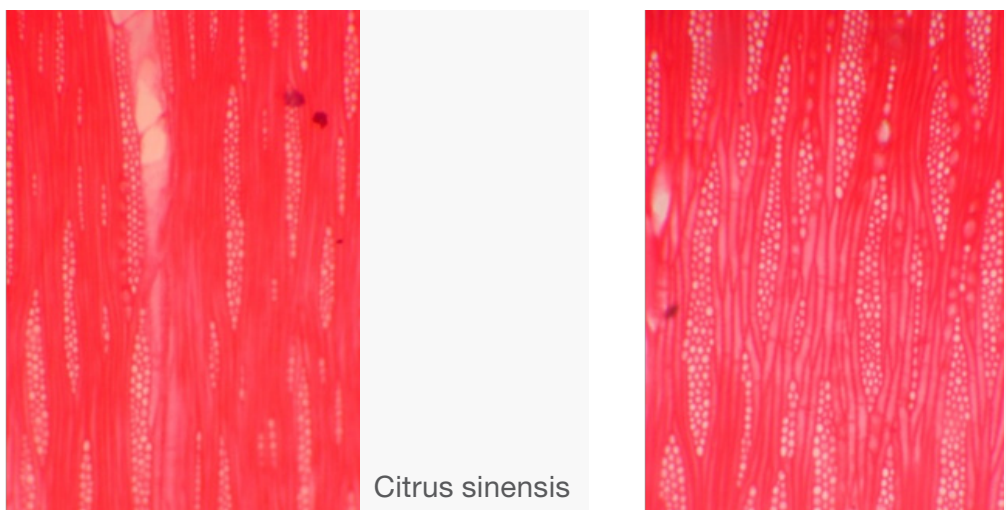

Citrus

sinensis var. Washington

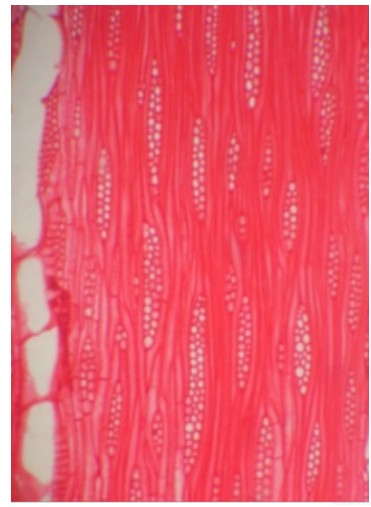

Citrus paradisi

Figura 4. Corte tangencial mostrando los radios observados en seis especies del género Citrus cultivadas en Costa Rica. 
Cuadro 2. Características de los vasos en la madera de seis especies del género Citrus cultivadas en Costa Rica.

\begin{tabular}{|c|c|c|c|c|c|c|}
\hline Característica & C. aurantifolia & C. limettioides & C. reticula & C. sinensis & $\begin{array}{l}\text { C. sinensis var. } \\
\text { Washington }\end{array}$ & C. paradisi \\
\hline Visibilidad & $\begin{array}{l}\text { Ligeramente } \\
\text { visibles a 10X }\end{array}$ & $\begin{array}{l}\text { Ligeramente } \\
\text { visibles a 10X }\end{array}$ & $\begin{array}{l}\text { Ligeramente } \\
\text { visibles a 10X }\end{array}$ & $\begin{array}{l}\text { Ligeramente } \\
\text { visibles a 10X }\end{array}$ & $\begin{array}{l}\text { Ligeramente } \\
\text { visibles a 10X }\end{array}$ & $\begin{array}{l}\text { Ligeramente visibles } \\
\text { a } 10 \mathrm{X}\end{array}$ \\
\hline Porosidad & Difusa & Difusa & Difusa & Difusa & Difusa & Visible a $10 \mathrm{X}$ \\
\hline Distribución & Cadenas radiales & $\begin{array}{l}\text { Bandas tangen- } \\
\text { ciales }\end{array}$ & $\begin{array}{l}\text { Bandas } \\
\text { tangenciales }\end{array}$ & $\begin{array}{l}\text { Tendencia a cade- } \\
\text { nas radiales }\end{array}$ & $\begin{array}{l}\text { Bandas tangen- } \\
\text { ciales }\end{array}$ & Difusa, patrón radial \\
\hline \multicolumn{7}{|l|}{$\begin{array}{l}\text { Agrupamiento de } \\
\text { vasos }\end{array}$} \\
\hline Solitarios (\%) & 77,46 & 41,51 & 35,27 & 37,44 & 54,84 & 95,68 \\
\hline $\begin{array}{l}\text { Múltiples de } \\
2 \text { a } 3(\%)\end{array}$ & 22,53 & 58,49 & 64,73 & 62,56 & 45,16 & 4,32 \\
\hline $\begin{array}{l}\text { Frecuencia (cant/ } \\
\mathrm{mm}^{2} \text { ) }\end{array}$ & $25,44(23,88-28,63)$ & $9,01(5,85-12,23)$ & $8,25(6,80-10,28)$ & $21,54(16,33-29,93)$ & $7,57(6,46-8,41)$ & $13,12(11,58-14,77)$ \\
\hline $\begin{array}{l}\text { Diámetro del lumen } \\
(\mu \mathrm{m})\end{array}$ & $62(48-82)$ & $58(30-75)$ & $53(36-71)$ & $61(27-90)$ & 110 (78-139) & $67(82-223)$ \\
\hline Largo $(\mu \mathrm{m})$ & $176(114-259)$ & 159 (100-230) & $149(59-225)$ & $167(116-254)$ & - & 943 (590-1260). \\
\hline Forma de sección & Redondeada & Redondeada & Redondeada & Redondeada & Redondeada & Redondeada \\
\hline Apéndices & Presentes & Presentes & Presentes & Presentes & Presentes & Presentes \\
\hline Tamaño & $\begin{array}{l}\text { Cortos }<1 / 3, \\
\text { ambos extremos }\end{array}$ & $\begin{array}{l}\text { Cortos }<1 / 3 \text {, en un } \\
\text { extremo }\end{array}$ & $\begin{array}{l}\text { Cortos }<1 / 3 \text {, en un } \\
\text { extremo }\end{array}$ & $\begin{array}{l}\text { Cortos }<1 / 3 \text {, en } \\
\text { ambos extremos }\end{array}$ & $\begin{array}{l}\text { Cortos }<1 / 3 \text {, en un } \\
\text { extremo }\end{array}$ & $\begin{array}{l}\text { Largos en ambos } \\
\text { extremos }\end{array}$ \\
\hline Gomas & Presentes & Presentes & Presentes & Presentes & Presentes & Presentes \\
\hline \multicolumn{7}{|l|}{$\begin{array}{l}\text { Punt. Inter- } \\
\text { vasculares }\end{array}$} \\
\hline Formas & $\begin{array}{l}\text { Hexagonales y } \\
\text { redondas }\end{array}$ & $\begin{array}{l}\text { Hexagonales y } \\
\text { redondas }\end{array}$ & $\begin{array}{l}\text { Hexagonales y } \\
\text { redondas }\end{array}$ & Poligonales & $\begin{array}{l}\text { Hexagonales y } \\
\text { redondas }\end{array}$ & $\begin{array}{l}\text { Hexagonales y } \\
\text { poligonales }\end{array}$ \\
\hline Distribución & Alternas & Alternas & Alternas & Alternas & Alternas & Alternas \\
\hline Abundancia & Media & Abundante & Escasa & Abundante & Abundante & Abundante \\
\hline $\begin{array}{l}\text { Diámetro promedio } \\
(\mu \mathrm{m})\end{array}$ & $3,16(2,36-4,42)$ & $1,63(0,78-2,34)$ & $1,58(1,04-2,47)$ & $4,16(2,88-4,84)$ & $2,36(1,92-2,74)$ & $5,00(2,73-9,12)$ \\
\hline Ornamentaciones & No presenta & No presenta & No presenta & No presenta & No presenta & No presenta \\
\hline $\begin{array}{l}\text { Canales } \\
\text { traumáticos }\end{array}$ & Presentes & Ausentes & Ausentes & Ausentes & Ausentes & Ausentes \\
\hline
\end{tabular}

Cuadro 3. Características de las fibras de seis especies del género Citrus cultivadas en Costa Rica.

\begin{tabular}{|c|c|c|c|c|c|c|}
\hline Característica & C. aurantifolia & C. limettioides & C. reticula & C. sinensis & $\begin{array}{l}\text { C. sinensis var. } \\
\text { Washington }\end{array}$ & C. paradisi \\
\hline $\begin{array}{l}\text { Espesor de pared } \\
(\mu \mathrm{m})\end{array}$ & $3,84(2,44-5,12)$ & $3,55(2,35-5,03)$ & $3,17(1,05-5,60)$ & $3,50(2,36-5,80)$ & $4,20(2,61-5,48)$ & $4,51(3,09-5,87)$ \\
\hline $\begin{array}{l}\text { Diámetro de fibra } \\
(\mu \mathrm{m})\end{array}$ & $15,31(10-22)$ & $12,38(9-15)$ & $13,78(10-17)$ & $14,98(11-20)$ & $13,56(8-20)$ & $17,06(13-24)$ \\
\hline $\begin{array}{l}\text { Diámetro de lumen } \\
(\mu \mathrm{m})\end{array}$ & $7,54(4,43-13,31)$ & $5,45(2,74-8,73)$ & $7,74(4,03-11,72)$ & $7,97(3,74-12,75)$ & $6,04(2,95-9,89)$ & $8,03(3,85-15,26)$ \\
\hline Largo de fibra $(\mu \mathrm{m})$ & $0,81(0,56-0,99)$ & $1,37(0,84-1,81)$ & $1,30(0,43-1.66)$ & $0,56(0,37-0,76)$ & $0,67(0,34-0,81)$ & $1,05(0,72-1,28)$ \\
\hline $\begin{array}{l}\text { Presencia de } \\
\text { septos }\end{array}$ & Ausentes & Ausentes & Ausentes & Ausentes & Ausentes & Ausentes \\
\hline \multicolumn{7}{|l|}{$\begin{array}{l}\text { Presencia de } \\
\text { cristales }\end{array}$} \\
\hline Tipo & Romboidales & Romboidales & Romboidales & Romboidales & Romboidales & Ausentes \\
\hline Abundancia & Escaso & Escaso & Escaso & Muy escaso & Muy escaso & - \\
\hline $\begin{array}{l}\text { Puntuaciones de } \\
\text { fibras }\end{array}$ & $\begin{array}{l}\text { Redondeada muy } \\
\text { pequeña }\end{array}$ & $\begin{array}{l}\text { Redondeada muy } \\
\text { pequeña }\end{array}$ & $\begin{array}{l}\text { Redondeada muy } \\
\text { pequeña }\end{array}$ & $\begin{array}{l}\text { Redondeada muy } \\
\text { pequeña }\end{array}$ & $\begin{array}{l}\text { Redondeada muy } \\
\text { pequeña }\end{array}$ & $\begin{array}{l}\text { Redondeada muy } \\
\text { pequeña }\end{array}$ \\
\hline
\end{tabular}


Cuadro 4. Características del parénquima axial de seis especies del género Citrus cultivadas en Costa Rica.

\begin{tabular}{|c|c|c|c|c|c|c|}
\hline Característica & C. aurantifolia & C. limettioides & C. reticula & C. sinensis & $\begin{array}{l}\text { C. sinensis var. } \\
\text { Washington }\end{array}$ & C. paradisi \\
\hline Tipo & $\begin{array}{l}\text { Paratraqueal y } \\
\text { apotraqueal }\end{array}$ & $\begin{array}{l}\text { Paratraqueal y } \\
\text { apotraqueal }\end{array}$ & $\begin{array}{l}\text { Paratraqueal y } \\
\text { apotraqueal }\end{array}$ & $\begin{array}{l}\text { Paratraqueal y } \\
\text { apotraqueal }\end{array}$ & $\begin{array}{l}\text { Paratraqueal y } \\
\text { apotraqueal }\end{array}$ & $\begin{array}{l}\text { Paratraqueal y } \\
\text { apotraqueal }\end{array}$ \\
\hline Visibilidad & Visible a 10X & Visible a 10X & Visible a $10 \mathrm{X}$ & Visible a $10 \mathrm{X}$ & Visible a 10X & Visible a 10X \\
\hline $\begin{array}{l}\text { Disposición de } \\
\text { paratraqueal }\end{array}$ & $\begin{array}{l}\text { Vasicéntrico escaso } \\
\text { y abundante, } \\
\text { aliforme y } \\
\text { confluente }\end{array}$ & $\begin{array}{l}\text { Vasicéntrico } \\
\text { escaso, confluente, } \\
\text { aliforme, unilateral }\end{array}$ & $\begin{array}{l}\text { Vasicéntrico } \\
\text { escaso, confluente, } \\
\text { unilateral } \\
\text { confluente. }\end{array}$ & $\begin{array}{l}\text { Vasicéntrico } \\
\text { escaso, unilateral } \\
\text { losangular } \\
\text { confluente de alas } \\
\text { cortas o largas }\end{array}$ & $\begin{array}{l}\text { Vasicéntrico } \\
\text { escaso, confluente } \\
\text { y unilateral }\end{array}$ & $\begin{array}{l}\text { Vasicéntrico, } \\
\text { confluente, aliforme } \\
\text { losangular, aliforme } \\
\text { en extensión lineal }\end{array}$ \\
\hline $\begin{array}{l}\text { Disposición de } \\
\text { apotraqueal }\end{array}$ & $\begin{array}{l}\text { Difuso agregados y } \\
\text { en líneas finas }\end{array}$ & $\begin{array}{l}\text { Difuso agregados } \\
\text { y en líneas finas de } \\
2-3 \text { filas }\end{array}$ & $\begin{array}{l}\text { Difuso agregados y } \\
\text { muy pocas líneas }\end{array}$ & $\begin{array}{l}\text { Difuso y muy pocas } \\
\text { líneas }\end{array}$ & $\begin{array}{l}\text { Difuso y bandas de } \\
3-4 \text { filas de ancho }\end{array}$ & $\begin{array}{l}\text { Bandas tangen- } \\
\text { ciales. }\end{array}$ \\
\hline Tipos de células & $\begin{array}{l}\text { Seriado de } 2 \\
\text { a } 3 \text { células por } \\
\text { filamento }\end{array}$ & $\begin{array}{l}\text { Seriado de } 2 \\
\text { a } 5 \text { células por } \\
\text { filamento }\end{array}$ & $\begin{array}{l}\text { Seriado de } 2 \\
\text { a } 3 \text { células por } \\
\text { filamento }\end{array}$ & $\begin{array}{l}\text { Seriado de } 2 \\
\text { a } 3 \text { células por } \\
\text { filamento }\end{array}$ & $\begin{array}{l}\text { Seriado de } 2 \\
\text { a } 3 \text { células por } \\
\text { filamento }\end{array}$ & $\begin{array}{l}\text { Fusiforme, de } 2 \\
\text { y algunas de } 3 \\
\text { a } 4 \text { células por } \\
\text { filamento }\end{array}$ \\
\hline Disposición & No estratificado & $\begin{array}{l}\text { No estratificado de } \\
2 \text { a } 3 \text { células }\end{array}$ & No estratificado & No estratificado & No estratificado & No estratificado \\
\hline \multicolumn{7}{|l|}{$\begin{array}{l}\text { Presencia de } \\
\text { cristales }\end{array}$} \\
\hline Tipo & Romboidales & Romboidales & Romboidales & Romboidales & Romboidales & Romboidales \\
\hline Abundancia & Escaso & Escaso & Escaso & Escaso & Escaso & Abundante \\
\hline $\begin{array}{l}\text { Punt. parénquimo- } \\
\text { vasculares }\end{array}$ & $\begin{array}{l}\text { Similar a las inter- } \\
\text { vasculares }\end{array}$ & $\begin{array}{l}\text { Similar a las inter- } \\
\text { vasculares }\end{array}$ & $\begin{array}{l}\text { Similar a las inter- } \\
\text { vasculares }\end{array}$ & $\begin{array}{l}\text { Similar a las inter- } \\
\text { vasculares }\end{array}$ & $\begin{array}{l}\text { Similar a las inter- } \\
\text { vasculares }\end{array}$ & $\begin{array}{l}\text { Similar a las inter- } \\
\text { vasculares }\end{array}$ \\
\hline Formas & $\begin{array}{l}\text { Simples con borde } \\
\text { distinto, redondas. }\end{array}$ & $\begin{array}{l}\text { Simples con borde } \\
\text { distinto, redondas. }\end{array}$ & $\begin{array}{l}\text { Simples con borde } \\
\text { distinto, redondas. }\end{array}$ & $\begin{array}{l}\text { Simples con borde } \\
\text { distinto, redondas. }\end{array}$ & $\begin{array}{l}\text { Simples con borde } \\
\text { distinto,redondas. }\end{array}$ & $\begin{array}{l}\text { Simples con borde } \\
\text { distinto,redondas. }\end{array}$ \\
\hline Distribución & Alternas & Alternas & Alternas & Alternas & Alternas & Alternas \\
\hline Abundancia & Media & Media & Media & Media & Media & Media \\
\hline $\begin{array}{l}\text { Diámetro promedio } \\
(\mu \mathrm{m})\end{array}$ & $2,44(1,48-3,38)$ & $2,03(1,18-2,86)$ & $2,20(1,69-2,80)$ & $3,63(2,63-, 81)$ & $2,07(1,18-3,15)$ & - \\
\hline Ornamentaciones & Ausentes & Ausentes & Ausentes & Ausentes & Ausentes & Ausentes \\
\hline
\end{tabular}

Cuadro 5. Características del parénquima radial de seis especies del género Citrus cultivadas en Costa Rica.

\begin{tabular}{|c|c|c|c|c|c|c|}
\hline Característica & C. aurantifolia & C. limettioides & C. reticula & C. sinensis & $\begin{array}{l}\text { C. sinensis var. } \\
\text { Washington }\end{array}$ & C. paradisi \\
\hline Visibilidad & $\begin{array}{l}\text { Ligeramente } \\
\text { visibles a 10X }\end{array}$ & Visibles a $10 \mathrm{X}$ & $\begin{array}{l}\text { Ligeramente } \\
\text { visibles a 10X }\end{array}$ & $\begin{array}{l}\text { Ligeramente } \\
\text { visibles a 10X }\end{array}$ & Visibles a 10X & $\begin{array}{l}\text { Ligeramente visi- } \\
\text { bles a simple vista }\end{array}$ \\
\hline Estructura & No estratificado & No estratificado & No estratificado & No estratificado & No estratificado & No estratificado \\
\hline Tipo & Multiseriados & Multiseriados & Multiseriados & Multiseriados & Multiseriados & Multiseriados \\
\hline Células de ancho & $2,30(1-3)$ & $2,44(1-4)$ & $2,04(1-3)$ & $2,00(1-3)$ & $2,80(1-4)$ & $4,45(3-7)$ \\
\hline Células en altura & $18,67(7-30)$ & $17,04(4-33)$ & $17,36(5-31)$ & $12,96(6-25)$ & $21,40(10-41)$ & $12,59(4-25)$ \\
\hline Composición & Homocelular & Homocelular & Homocelular & Homocelular & Homocelular & Homocelular \\
\hline \multicolumn{7}{|l|}{ Tipo de células } \\
\hline Arriba & Procumbentes & Procumbentes & Procumbentes & Procumbentes & Procumbentes & Procumbentes \\
\hline Cuerpo de Radios & Procumbentes & Procumbentes & Procumbentes & Procumbentes & Procumbentes & Procumbentes \\
\hline Abajo & Procumbentes & Procumbentes & Procumbentes & Procumbentes & Procumbentes & Procumbentes \\
\hline $\begin{array}{l}\text { Presencia de } \\
\text { cristales }\end{array}$ & Ausentes & Ausentes & Ausentes & Ausentes & Ausentes & Ausentes \\
\hline Ancho $(\mu \mathrm{m})$ & $\begin{array}{l}21,09 \\
(7,83-27,04)\end{array}$ & $\begin{array}{l}20,33 \\
(7,69-32,05)\end{array}$ & $\begin{array}{l}17,29 \\
(5,13-26,92)\end{array}$ & $\begin{array}{l}24,13 \\
(9,06-43,54)\end{array}$ & $\begin{array}{l}37,12 \\
(13,33-47,66)\end{array}$ & $\begin{array}{l}46,56 \\
(27,59-65,52)\end{array}$ \\
\hline Altura $(\mu \mathrm{m})$ & $214(78-408)$ & $193(56-343)$ & $187(52-336)$ & $196(59-355)$ & $250(106-431)$ & $267(100-524)$ \\
\hline
\end{tabular}


Cuadro 5. Características del parénquima radial de seis especies del género Citrus cultivadas en Costa Rica.

\begin{tabular}{|c|c|c|c|c|c|c|}
\hline Característica & C. aurantifolia & C. limettioides & C. reticula & C. sinensis & $\begin{array}{l}\text { C. sinensis var. } \\
\text { Washington }\end{array}$ & C. paradisi \\
\hline $\begin{array}{l}\text { Frecuencia } \\
\text { (cant/mm²) }\end{array}$ & $4,25(3,28-4,92)$ & $3,83(2,87-5,33)$ & $3,72(2,75-4,47)$ & $3,17(2,00-4,00)$ & $3,72(2,37-4,74)$ & $4,38(2,62-5,98)$ \\
\hline \multicolumn{7}{|l|}{$\begin{array}{l}\text { Punt. radio- } \\
\text { vasculares }\end{array}$} \\
\hline Formas & $\begin{array}{l}\text { Redondeadas, } \\
\text { poligonales y } \\
\text { rectangulares }\end{array}$ & $\begin{array}{l}\text { Rectangulares y } \\
\text { ovaladas }\end{array}$ & $\begin{array}{l}\text { Rectangulares y } \\
\text { ovaladas }\end{array}$ & $\begin{array}{l}\text { Redondeadas y } \\
\text { ovaladas }\end{array}$ & $\begin{array}{l}\text { Rectangulares, } \\
\text { ovaladas y } \\
\text { poligonales }\end{array}$ & $\begin{array}{l}\text { Rectangulares, } \\
\text { ovaladas y } \\
\text { poligonales }\end{array}$ \\
\hline Distribución & Alternas & Alternas y opuestas & Alternas y opuestas & Alternas & Alternas y opuestas & Alternas y opuestas \\
\hline Abundancia & Media & Abundante & Abundante & Abundante & Abundante & Abundante \\
\hline $\begin{array}{l}\text { Diámetro promedio } \\
(\mu \mathrm{m})\end{array}$ & $2,51(1,28-3,54)$ & $1,83(1,30-2,34)$ & $2,13(1,43-2,60)$ & $3,31(2,15-4,75)$ & $1,79(1,28-2,37)$ & $2,79(2,28-3,37)$ \\
\hline Ornamentaciones & Ausente & Ausente & Ausente & Ausente & Ausente & Ausente \\
\hline
\end{tabular}

La presencia de canales traumáticos estuvo presente solamente en $C$. aurantifolia, permitiendo ser la característica principal para su identificación.

Con respecto a la anatomía microscópica, se presentan pocas diferencias entre las especies evaluadas, entre ellas: aunque se observó una tendencia a formar anillos de crecimiento, fue frecuente encontrar la presencia de anillos falsos en las seis especies de Citrus en estudio, C. paradisi posee la mayor cantidad de poros solitarios, C. aurantifolia, C. sinensis y C. sinensis var. Washington presentaron gran cantidad de sustancias en vasos, solo en C. paradisi no se observó cristales presentes, $C$. sinensis var. Washington presentó la menor cantidad de parénquima apotraqueal, en C. sinensis var. Washington, los radios fueron ligeramente más anchos y más largos que el resto de las especies, en $C$. sinensis los radios fueron más pequeños en comparación a los presentados en el resto de las especies.

\section{Agradecimientos}

A la Vicerrectoría de Investigación y Extensión del Instituto Tecnológico de Costa Rica por el apoyo financiero al Proyecto de Investigación. A los propietarios y personal de los cafetales donde fueron extraídas las muestras de los árboles. A Iriabelle González Leal y Manuel Morales Salazar por la información suministrada para una de las especies.

\section{Referencias}

Abrems, MD; Hock, WK. 2006. Annual growth rings and the impact of Benlate 50 DF fungicide on Citrus trees in seasonally dry tropical plantations of northern Costa Rica. Forest Ecology and Management 227 (1-2): 96-101.
Brenes, LE; Arauz, LE. 1994. Crop protection in Citrus production. Pesticide use in Costa Rica's Northem region orchards. Preliminary report Interlinkage Program NCSUUNA-UCR San José, Costa Rica. sp.

COPANT (Comisión Pan-Americana de Normas Técnicas, BO). 1974 Descripción macroscópica, microscópica y general de la madera (Esquema 1). COPANT 30:1-19.

Corrales, G. 1997. Proyecto de manejo y tratamiento de cáscaras de naranja provenientes de la planta procesadora Del Oro S. A. Evaluación del sitio dentro del Área de Conservación Guanacaste (Sector El Hacha), destinado a recibir las cáscaras de naranja. ACG, Costa Rica. 59 p.

Espinoza, N; León, W. 2001. Anatomía de la madera. Universidad de Los Andes, Consejo de Publicaciones y Consejo de Desarrollo Científico, Humanístico y Tecnológico. Mérida, Venezuela. $397 \mathrm{p}$.

IAWA (International Association of Wood Anatomy, BE). 1989. List of microscopic features for hardwood identification. IAWA Bulletin 10 (3): 226-332.

IBAMA (Instituto Brasileiro do Meio Ambiente e dos Recursos Naturais Renováveis). 1992. Normas e procedimentos em estudos de anatomia da madeira: angiospermas e gimnospermas. Brasília: Diretoria de Incentivo à Pesquisa e Divulgação (Série Técnica 15). 17 p.

Johansen, DA. 1940. Plant microtechnique. New York. MacGraw-Hill. 533 p.

Mattos, DJ; Quaggio, JA; Cantarella, H; Kumar, A. 2003. Nutrient content of biomass components of Hamlin sweet orange trees. Scientia Agricola 60 (1): 155-160.

Molina, E. 1999. Fertilización y nutrición de naranja en Costa Rica. (XI Congreso Nacional Agronómico / III Congreso Nacional de Suelos. San José, Costa Rica del 19 al 23 de julio de 1999, San José, CR). Memorias. San José, CR, El Congreso.

Sánchez, C. 2005. Producción y Comercialización de Cítricos. Lima. Ediciones Ripalme. pp: 25-29; 110, 111.

Sass, JE. 1951. Botanical microtechnique. 2 ed. lowa. State College Press. 228 p. 